\title{
INTRODUCTION A L'OVOGENÈSE CHEZ LES INVERTÉBRÉS
}

\author{
Hélène CHARNIAUX-COTTON \\ Laboratoire de Sexualité et Reproduction des Invertébrés, \\ Tour 32, Université Paris VI, \\ 4, Place Jussieu, 75230 Paris Cédex 05 (France)
}

\section{RÉSUMÉ}

L'ovogenèse chez les Invertébrés, comme chez les Vertébrés, peut se diviser en trois étapes ; la prophase de méiose avec chromosomes visibles; la première croissance (ou prévitellogenèse); la vitellogenèse. Chez divers Invertébrés il a été prouvé expérimentalement que les ovogonies évoluent spontanément jusqu"à la fin de la prévitellogenèse (" autodifférenciation ovarienne "). La vitellogenèse nécessite au contraire l'intervention d'un facteur d'origine nerveuse.

Pour illustrer les données acquises chez les Invertébrés, trois exemples sont exposés : les Hydres, les Céphalopodes et les Étoiles de mer.

Chez les Hydres, les individus élevés dans de bonnes conditions sont dépourvus de cellules germinales. Celles-ci se forment à partir des cellules interstitielles quand les conditions externes deviennent défavorables. L'ovogenèse s'effectue ensuite sans blocage. Des expériences ont démontré que l'ovogenèse se réalise quand la neurosécrétion, facteur positif de croissance, a disparu.

Chez les Mollusques Céphalopodes existe une paire de glandes endocrines : les glandes optiques, qui sécrètent un facteur gonadotrope déclenchant la vitellogenèse. L'activité de chacune de ces glandes est inhibée par un nerf provenant du lobe sous-pédonculé du cerveau. Ce contrôle inhibiteur cérébral est activé par la lumière et levé par l'obscurité.

Chez les Étoiles de mer (Échinodermes) a été isolée, pour la première fois, une substance responsable de la maturation des ovocytes (MIS) : la r-méthyladénine. La MIS est synthétisée par l'ovaire sous le contrôle d'un facteur gonadotrope, de nature polypeptidique, sécrété par les nerfs radiaux : la GSS.

L'ovogenèse présente les mêmes phénomènes cytologiques chez les Invertébrés et les Vertébrés :

- mitoses ovogoniales,

- entrée en prophase de méiose,

- blocage de cette prophase au stade diplotène ou diacinèse,

- croissance de l'ovocyte divisée généralement en :

r. une première croissance ou prévitellogenèse caractérisée par d'importantes synthèses d'ARN ribosomiaux et messagers,

2. une vitellogenèse correspondant à l'accumulation de lipides, glucides et protéines endogènes et/ou exogènes,

- reprise de la méiose, 
- nouveau blocage de la méiose à un stade variable, les cas extrêmes étant la réalisation totale de la méiose (ex. : Echinodermes) ou aucune reprise de la méiose (ex. : Ascaris, Insectes) avant la fécondation,

- fécondation,

- fin de la méiose.

Chez les Invertébrés suivants, il a été prouvé expérimentalement que l'ovogenèse, tout au moins jusqu'à la fin de la prévitellogenèse, est l'évolution spontanée des gonies en l'absence d'un inducteur ou d'une hormone androgène :

- les Crustacés supérieurs (Charniaux-Corton, I959, I972),

- 1'Insecte Lampyris noctiluca (NAISSE, I966),

- les Hydres (voir BRIEN, I972),

- le Myriapode Chilopode Lithobius forficatus (ZERBIB, I966),

- les Mollusques gastéropodes hermaphrodites : Calyptraea sinensis (STREIFF, I967), Helix aspersa (GuYARD, I969),

- l'Oligochète Eisenia foetida, également hermaphrodite (LATTAUD, I973).

Cette autodifférenciation ovocytaire explique la présence fréquente chez les Invertébrés d'ovogenèse dans les testicules, la situation inverse n'étant pas observée : en effet, chez un mâle, des gonies peuvent échapper au facteur masculinisant; chez une femelle, celui-ci n'existe pas et la spermatogenèse est impossible.

Chez les Invertébrés comme chez les Vertébrés les facteurs externes contrôlent la reproduction puisque celle-ci ne s'effectue que dans certaines conditions externes. Les nombreux résultats obtenus montrent que les facteurs externes agissent par l'intermédiaire de la neurosécrétion laquelle contrôle le déroulement de 1'ovogenèse au niveau d'un certain stade qui est le plus souvent l'entrée en vitellogenèse. Cependant chez les Hydres et probablement chez les Annélides Polychètes, ce contrôle s'exerce au niveau de la formation des ovogonies.

La neurosécrétion agit le plus souvent directement sur la gonade. Chez les Mollusques Céphalopodes, les Insectes, elle contrôle l'activité d'une glande endocrine source du facteur gonadotrope.

Pour compléter les trois communications relatives aux Annélides Polychètes, Crustacés et Insectes, je traiterai brièvement les Hydres, les Mollusques Céphalopodes et les Échinodermes. Chez ces derniers a été isolée la substance responsable de la maturation des ovocytes; il s'agit d'un résultat remarquable puisque c'est le premier obtenu chez les Invertébrés et Vertébrés.

\section{LES HYDRES}

Elevées au laboratoire à $20^{\circ}$ et dans de bonnes conditions Hydra fusca ne possède pas de cellules germinales et se reproduit par bourgeonnement. Les cellules interstitielles, cellules peu différenciées, participent activement au renouvellement cellulaire, particulièrement à celui des cnidoblastes.

Lorsque 1'Hydre est placée à $8^{\circ}$ (BRIEN, I966), les cellules interstitielles se transforment en ovogonies chez les femelles (en spermatogonies chez les mâles) et l'ovogenèse se déroule sans blocage jusqu'à la formation de l'œuf. Les Hydres sont donc comme des plantes : les conditions externes induisent la reproduction sexuée. 
BURNETT et DiEHI, (I964) ont démontré expérimentalement que ces facteurs externes agissent par l'intermédiaire de cellules neurosécrétrices. A $20^{\circ}$, ces cellules synthétisent un facteur de croissance inhibiteur de la formation des gonies. A $8^{\circ}$, elles cessent leur activité et les cellules interstitielles se transforment en cellules germinales. Des expériences réalisées par BRIEN (I963) prouvent que cette transformation nécessite en outre l'intervention d'un facteur positif. Cet auteur a obtenu chez Hydra fusca des individus qui deviennent spontanément et définitivement incapables de former des gonies à 80; il les a qualifiés d'asexués. Des parabioses sont réalisées entre une hydre asexuée et une hydre normale en les accolant endoderme contre endoderme après incision longitudinale. Dans les chimères ainsi obtenues et portées à $8^{\circ}$, une gamétogenèse se manifeste d'abord sur la face de la colonne correspondant à l'hydre normale ; elle s'étend ensuite à la face correspondant à l'hydre asexuée, toujours à partir des deux lignes de suture (fig. I).

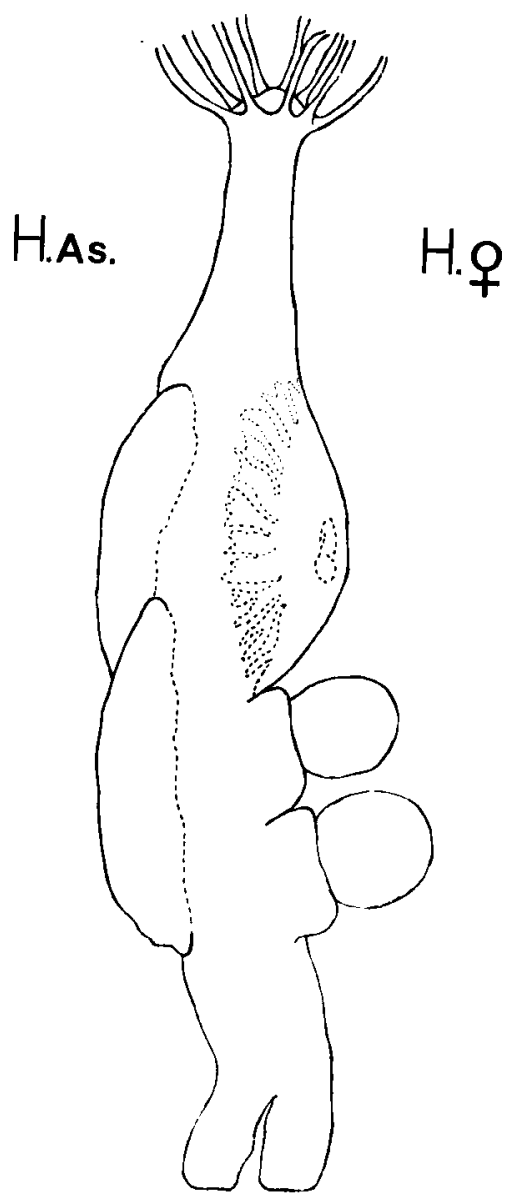

Fic. I. - Aspect de la greffe en parabiose entre Hydra fusca asexué (H. As.) et Hydra fusca sexuée femelle (H. + ) 25 jours après la mise en gamétogenèse à $8^{\circ}$

La face normale (H.ㅇ) porte des enflures ovariques et déjà des oufs éclos. La face asexuée (H. As.) porte des enflures ovariques contenant chacune un ovocyte achevant sa croissance (BRIEN, I966). 
Je pense que l'on peut déduire de ces travaux la conclusion suivante : le facteur responsable de la transformation des cellules interstitielles en gonies n'est synthétisé qu'en l'absence de neurosécrétion; ajoutons qu'il est regrettable qu'aucune étude des cellules neurosécrétrices chez les hydres asexuées n'ait encore été réalisée.

En ce qui concerne le contrôle du sexe des gonies, BRIEN (1963) a démontré qu'il existe un facteur masculinisant; en son absence, les gonies évoluent en ovogonies (autodifférenciation ovocytaire) : en effet, dans des parabioses entre un mâle et une femelle, la femelle est toujours masculinisée. Ce contrôle du sexe explique que dans les parabioses entre individus normaux et asexués, la gamétogenèse chez ces derniers est du sexe de l'hydre inductrice.

En conclusion, les travaux réalisés chez les Hydres montrent l'universalité de la neurosécrétion et celle de son rôle dans la reproduction ; ils mettent bien en évidence l'intervention de deux contrôles dans la différenciation des cellules germinales : celui de la différenciation de cellules en gonies (induction germinale) et celui du sexe (orientation des gonies en ovogonies ou spermatogonies). Enfin, chez les Hydres, l'activité génitale est contrôlé au niveau de la formation des gonies : les ovogonies une fois formées, l'ovogenèse se déroule sans blocage. Une telle situation existe probablement dans d'autres groupes.

\section{ILES MOILUUSQUES CÉPHALOPODES}

Il existe chez les Mollusques Céphalopodes une paire de glandes appelées optiques accolées aux pédoncules des ganglions optiques. Elles reçoivent un nerf issu d'une région postérieure du cerveau (le lobe sous-pédonculé). Elles sont constituées par des cellules étoilées contenant un matériel dense aux électrons et des cellules de soutien. Les fibres nerveuses se terminent contre les cellules étoilées et exercent sur elles un effet inhibiteur (NISHIOKA, BERN et GoldING, 1970). En effet, chez la Pieuvre femelle immature, lorsque le nerf est sectionné, les glandes optiques augmentent de volume et libèrent un facteur gonadrope qui déclenche la vitellogenèse. Le même résultat est obtenu par la destruction d'un lobe sous-pédonculé. La section des voies optiques périphériques provoque également la vitellogenèse. Enfin, après l'excision des glandes, il n'y a jamais de vitellogenèse quel que soit le type d'intervention ce qui prouve leur fonction gonadotrope (WELLS et WELLS, I959). La région souspédonculée est donc activée par la lumière et elle inhibe la sécrétion du facteur gonadotrope par les glandes optiques (fig. 2). Ces résultats expliquent que durant la vitellogenèse, les Pieuvres et les Seiches se tiennent à l'obscurité dans les fonds marins.

La culture organotypique d'ovaires de Seiche (RICHARD, I97I) a confirmé tous les résultats opératoires. Les glandes optiques cultivées seules, donc libérées de l'inhibition cérébrale, montrent une grande activité sécrétrice. Leur association à des ovaires permet de préciser qu'elles déclenchent la vitellogenèse seulement dans les ovocytes ayant terminé leur première croissance. Cette dernière s'effectue dans des ovaires cultivés seuls, donc en l'absence de tout contrôle hormonal. 

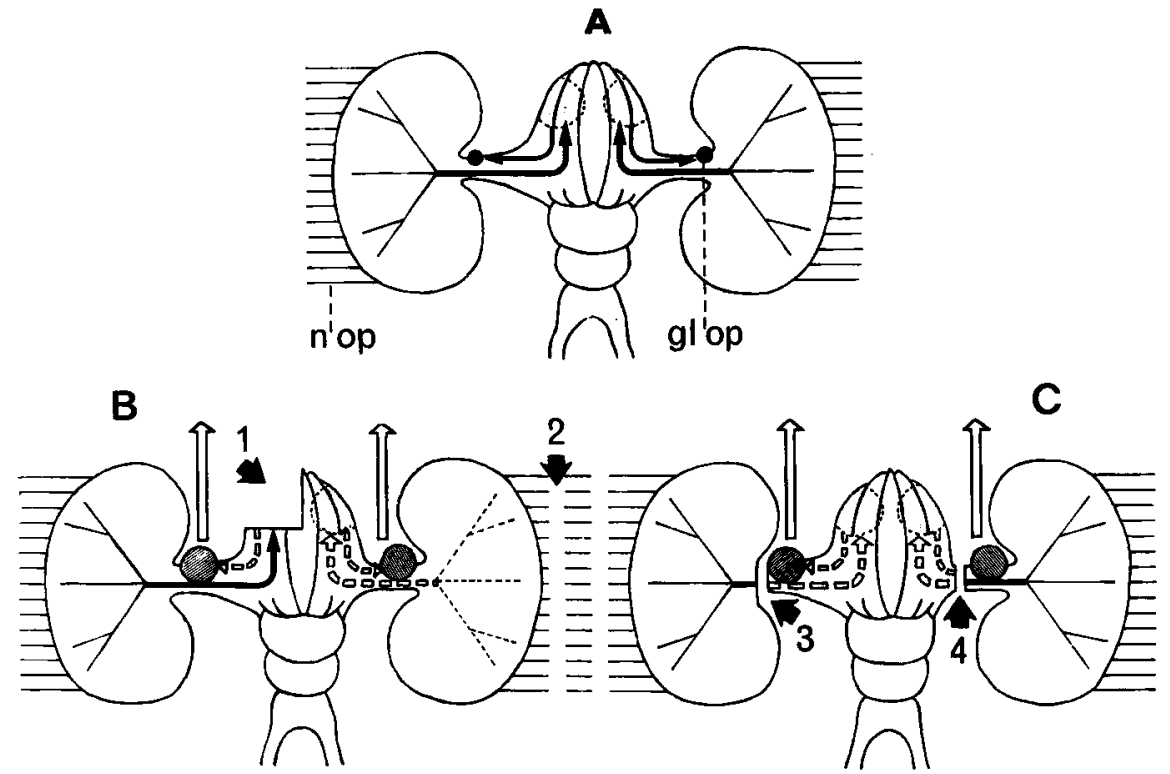

FIG. 2. - Contróle hormonal de la maturation génitale chez Octopus

A : chez l'individu immature l'activité de la glande optique est inhibée par voie nerveuse; B et C : maturation expérimentale déterminée par : $\mathrm{I}^{\circ}$ ablation du centre nerveux inhibiteur; $2^{\circ}$ section du nerf optique ; $3^{\circ}$ ablation du lobe optique $; 4^{\circ}$ section du tractus optique. gl.op. : glande optique ; n.op. : nerf optique. (D'après Wells et WeLLS, r959.)

\section{LES ÉCHINODERMES}

Les Etoiles de mer ont fait l'objet de recherches qui ont permis - pour la première fois dans le règne animal - l'isolement d'une substance responsable de la maturation des ovocytes: la I-méthyladénine (KANATANI et al., I969).

Il avait d'abord été observé que les extraits de nerfs radiaux provoquent la ponte et la maturation (disparition de la vésicule germinative et reprise de la méiose) des ovocytes (voir KANATANI, I972). La purification de la substance gonado-stimulante (GSS) a été réalisée à partir de nerfs radiaux d'Asterias amurensis par KANATANI. Il s'agit d'un polypeptide constitué par douze acides aminés : acide aspartique (2), thréonine (r), sérine (6 ou 7 ), acide glutamique (I), proline (I), glycine (4), alanine (2), valine (I), isoleucine (I), leucine (I), histidine (I), ornithine (I). La GSS agit à une concentration de $0,0096 \mu \mathrm{g} / \mathrm{ml}$. Elle est identique chez le mâle et la femelle. Elle présente une certaine spécificité.

Kanatani a observé que des ovocytes prélevés dans l'ovaire et mis dans l'eau de mer en présence de GSS ne présentent aucune reprise de la méiose. Celle-ci a lieu si on ajoute un fragment ovarien; donc, sous l'influence de la GSS, ce fragment a produit une substance induisant la méiose (MIS). Cette substance a été isolée à partir de $20 \mathrm{~kg}$ d'ovaires d'Asterina pectinifera; il s'agit de la I-méthyladénine. Elle est active à la concentration de $3 \times \mathrm{IO}^{-7} \mathrm{M}$. Elle n'est pas spécifique.

Le lieu de synthèse serait les follicules des ovocytes. En effet, si on met un grand nombre d'ovocytes dans de l'eau de mer avec de la GSS, on obtient la matu- 
ration. Dans les premières expériences, KATANI n'avait mis que quelques ovocytes dans l'eau de mer contenant de la GSS ; il est probable que la petite quantité de MIS formée s'est dissoute.

La MIS n'agit que si elle est appliquée à l'extérieur de l'ovocyte. Injectée directement dans la cellule femelle, elle ne provoque pas la disparition de la vésicule germinative. La MIS induit donc la production d'un troisième messager dans le cortex de l'œuf et qui serait le véritable inducteur de la maturation. (fig. 3)

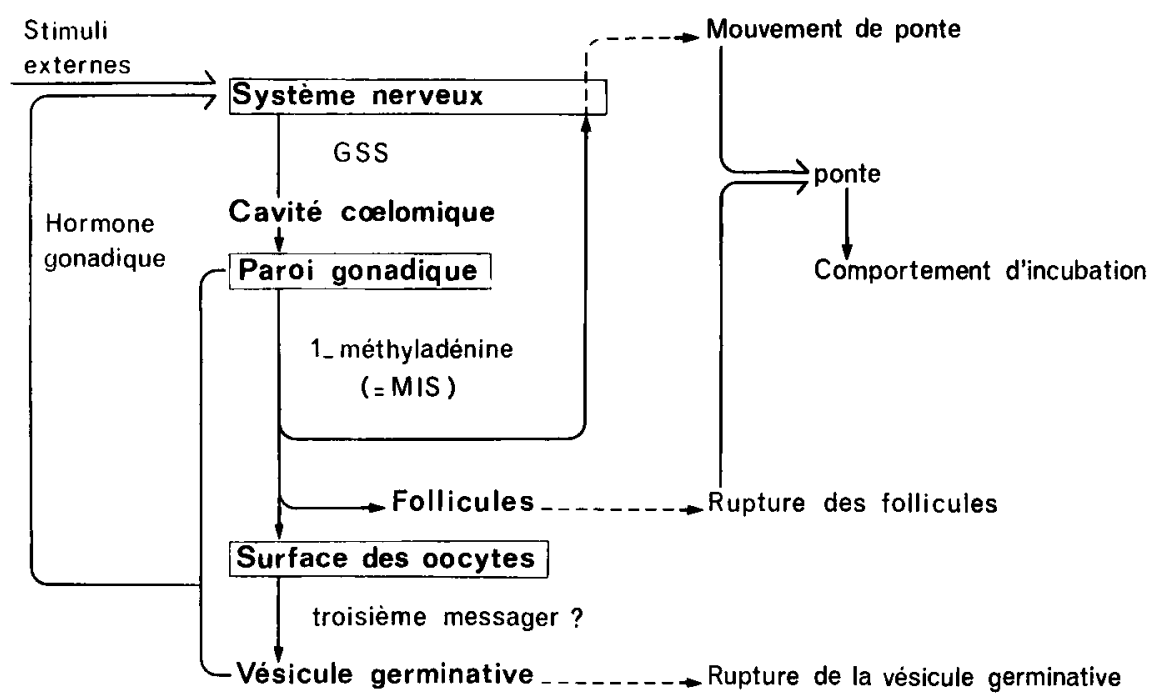

FIg. 3. - Contrôle de la maturation et de la ponte des ovocytes chez l'étoile de mer GSS : gonad stimulating substance; MIS : meiosis inducing substance

(D'après Kanatani, r972)

\section{SUMMARY}

\section{INTRODUCTION TO OOGENESIS IN INVERTEBRATES}

As in vertebrates, oogenesis in invertebrates may be divided into three stages : prophase of meiosis with visible chromosomes, previtellogenesis (or first growth) and vitellogenesis. In several groups of invertebrates, it was experimentally proved that oogonia spontaneously accomplish oogenesis up to the end of previtellogenesis ("ovarian self-differentiation "). Vitellogenesis, on the other hand, needs a factor from the nervous system.

For illustrating the various modalities of oogenesis in invertebrates, three samples are used : hydrae, cephalopoda and starfish.

Hydrae specimens kept in good conditions do not possess germinal cells. When conditions become unfavorable, oogonia are differentiated from the interstitial cells. These oogonia accomplish oogenesis up to female gametes with no resting stage. Experiments have proved that oogenesis occurs when neurosecretion, which is a positive growth factor, disappears.

In cephalopod mollusca, vitellogenesis is regulated by the secretion of a pair of endocrine glands, the optic glands. The activity of each gland is inhibited by a nerve originating in the subpedunculate lobe of the brain. This inhibitory control is activated by light and suppressed by darkness.

I-methyladenine (MIS), a substance responsible for oocyte maturation, is isolated and identified for the first time in starfish. The MIS is synthesized by the ovary under the control of a gonadotrophic factor, GSS, which is a polypeptide secreted by the radial nerves. 


\section{RÉFÉRENCES BIBLIOGRAPHIQUES}

Brien P., r963. Contribution à l'étude de la biologie sexuelle chez les Hydres d'eau douce. Induction gamétique et sexuelle par la méthode des greffes en parabiose. Bull. Biol. France-Belgique, 97, 214283.

BrIen P., 1966. Biologie de la reproduction animale. Collection « Les grands problèmes de la biologie 》, direction P.-P. Grassé, Masson et Cle, Éditeurs, Paris. 292 p.

Brien P., 1972. Influence humorale dans la différenciation sexuelle chez les Hydraires. In Cours et documents de Biologie, 3 : Hormones et différenciation sexuelle chez les Invertébrés, direction E. Wolff, Gordon et Breach, Editeurs, Paris Londres, N. Y., p. I-43-

Burnetr A. L., Dienl N. A., I964. The nervous system of Hydra III. The initiation of sexuality with special reference to the nervous system. J. exp. Zool., 157, 237-250.

Charniaux-Cotton H., 1959. Étude comparée du développement postembryonnaire de l'appareil génital et de la glande androgène chez Orchestia gammarella et Orchestia mediterranea (Crustacés Amphipodes). Autodifférenciation ovarienne. Bull. Soc. Zool., 84, ro5-1 5.

Charniaux-Cotron H., I972. Recherches récentes sur la différenciation sexuelle et l'activité génitale chez divers Crustacés supérieurs. In Cours et documents de Biologie, 3 : Hormones et différenciation sexuelle chez les Invertebrếs, direction E. Wolff; Gordon et Breach, Éditeurs, Paris, Londres, N. Y., 127-I 78 .

GuYARD A., r969. Autodifférenciation femelle de l'ébauche gonadique de l'Escargot Helix aspera müLL. cultivée sur milieu anhormonal, C. R. Acad. Sci. Fr., 268, 966-969.

Kanatani H., 1972. Nature chimique et mode d'action de la substance inductrice de la méiose chez l'Étoile de mer : étude de la ponte et de la maturation des ovocytes. In Cours et documents de Biologie, 3.: Hormones et différenciation sexuelle chez les Invertébrés, direction $\mathbf{E}$. Wolff; Gordon et Breach, Éditeurs, Paris, Londres, N. Y., 245-262.

Kanatani H., Shirai H., Nakanishi K., Kurokawa T., ig69. Isolation and identification of meiosis inducing substance in starfish Asterias amurensis. Nature, 221, 273-274.

Lattaud C., I973. Preuve expérimentale de l'autodifférenciation ovarienne chez l'Annélide Oligochète Eisenia foetida f. typica sav. C. R. Acad. Sci. Fr., D, 276 I737-r74o.

NaIsse J., I966. Contrôle endocrinien de la différenciation sexuelle chez Lampyris noctiluca. III. Influence des hormones de la pars intercerebralis. Gen. Comp. Endocrinol., 7, ro5-1 Io.

Nishioka R. S., Bern H. A., Golding D. W., r97o. Innervation of the Cephalopod Optic Gland. In Aspects of Neuroendocrinology. Direction W. Bargmann et Sharrer, Springer-Verlag, Berlin, Heidelberg, N. Y., 49-54.

Richard A., I97I. Différenciation sexuelle des Céphalopodes en culture in vitro. In Les apports récents des cultures et des greffes en biologie animale, Masson et Cie Éditeurs, Paris, I69-I75.

StreifF W., 1967. Étude endocrinologique du déterminisme du cycle sexuel chez un Moliusque hermaphrodite protandre Calyptraea sinensis L. III. Mise en évidence, par culture in vitro, de facteurs conditionnant l'évolution de la gonade. Ann. Endocrinol. Paris, 28, 64 1-656.

Wells M. J., Wells J., r959. Hormonal control of sexual maturity in Octopus. J. Exp. Biol., 36, I-33.

ZeRBiB C., I966. Étude descriptive et expérimentale de la différenciation de l'appareil génital du Myriapode Chilopode Lithobius forficatus L. Bull. Soc. zool. Fr., 91, 203-216. 